\title{
Programa combinado para mejorar el estado emocional y prevenir el deterioro cognitivo de adultos mayores institucionalizados
}

\author{
María Begoña Garín Gómez \\ Estudiante de doctorado de la Facultad de Psicología, Universidad Nacional de Educación a Distancia, \\ Alfredo Briones Aranda \\ Profesor investigador de la Facultad de Medicina Humana, Universidad Autónoma de Chiapas, \\ Soledad Ballesteros Jiménez \\ Profesora titular de la Facultad de Psicología, Universidad Nacional de Educación a Distancia,
}

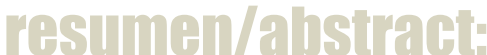

Diversos programas de entrenamiento combinado (PEC) basados en ejercicios físicos y neuropsicológicos se utilizan cada vez más con mejores resultados, dentro de las terapias no farmacológicas. En el presente trabajo, se realizó un estudio empírico cuasi-experimental, en donde se evaluó la influencia de un PEC sobre la mejora de las capacidades afectivas, cognitivas y de independencia, tras un año de intervención con adultos mayores institucionalizados (AMI) de una

residencia para ancianos en Tuxtla Gutiérrez Chiapas, México (19 mujeres y 23 hombres).

Durante este proceso, tanto al inicio como al final, se evaluaron las distintas áreas de estudio, mediante la utilización de las pruebas de Hamilton, Beck, Lobo y Barthel, en donde se pudo observar una tendencia positiva ejercida por el PEC en la disminución de los niveles de ansiedad y depresión en los hombres, así como la disposición que tuvo este programa para evitar el progreso del deterioro cognitivo en ambos grupos. Por lo anterior y en consideración de la carencia de programas destinados

a coadyuvar el mejoramiento de la salud mental de los AM en el estado de Chiapas, el PEC podría proponerse como una estrategia de bajo costo, encaminada a procurar la salud integral de los AMI.

It is ever more common to use diverse programs of combined training (PCT) based on physical and neuropsychological exercise to improve the results of non-pharmacological therapies. However, there is a scarcity of such programs used as coadjuvant therapy for improving the mental health of seniors

in the state of Chiapas, Mexico. In the present study, a quasi-experimental empirical study was carried out to evaluate the influence of a one-year PCT on the improvement of capacities related to affection, cognition and independence. The program was applied to seniors (19 women and 23 men) institutionalized in a home in Tuxtla Gutierrez, Chiapas, Mexico.

The tests of Hamilton, Beck, Lobo and Barthel were employed at the beginning and end of the one-year program to evaluate various areas. At the end of the program, there was a tendency to a decrease in the anxiety and depression of the men, as well as in the cognitive deterioration of both groups. Hence, this

PCT could be a useful and low-cost strategy aimed at fortifying the overall health of seniors.

\section{palabras clave/keywords:}

Ansiedad, depresión, deterioro cognitivo, estrés, dependencia, adulto mayor Anxiety, depression, cognitive impairment, stress, dependence, older adult. 


\section{Introducción}

Recientemente, el grupo de los adultos mayores (AM) ha cobrado mayor relevancia en la distribución de la pirámide poblacional mexicana. En el estado de Chiapas los AM representan $4.9 \%$ de la población, lo que equivale a 234982 adultos, mayores de 65 años (censo de población y vivienda, 2010). En el panorama mundial, este grupo etario se ha relacionado con un incremento en las enfermedades mentales, entre las que se incluyen los trastornos emocionales (depresión y ansiedad) y los trastornos cognitivos como la enfermedad de Alzheimer (EA) (Ballesteros y Reales, 2004; Ballesteros, Reales, Mayas, y Heller, 2008).

En los AM, la enfermedad afectiva más frecuente y que se acentúa con mayor severidad, es la depresión, influenciada por factores psicosociales, como el deterioro en la salud, percepción de indefensión, duelos, problemas económicos, abandono familiar, y otros (Pérez, 2006; Chou y Cheung, 2013; Feola y cols., 2013).

La ansiedad puede definirse como una respuesta disfuncional y adaptativa frente a situaciones potencialmente peligrosas, la cual puede incidir negativamente en el proceso de envejecimiento (DSM-IV TR) (Grenier y cols., 2011). Algunos factores de riesgo en los AM para desarrollar este trastorno, son: el género -ser mujer-, status de viudez, la jubilación, enfermedades, disfunción familiar, etc. (Eaton y Keyl, 1990; Feola y cols., 2013).

La pérdida de memoria es uno de los aspectos principales del deterioro cognitivo que ocurre durante el envejecimiento (Light, 1991; Hogervorst, 2013). La disminución en la tasa de fluidez verbal categorial, es uno de los indicios o síntomas que prime- ro aparecen como parte de las alteraciones en la memoria semántica en las demencias tipo EA, alteración que es tanto un buen predictor cognitivo como un indicador del grado de severidad de las demencias (Peraita y Sánchez Bernardos, 1998; Peraita y Moreno, 2006).

Existen diversos factores que pueden estar asociados a las alteraciones de memoria a una edad avanzada, los cuales los hemos agrupado en dos grandes rubros, las enfermedades crónicas degenerativas y factores o trastornos neuropsicológicos estrechamente vinculados al primer rubro. Como ejemplos relacionados con las enfermedades crónicas se describen, la diabetes mellitus (Rao, 2013), la hipertensión arterial (Alosco y cols., 2012), artritis reumatoide (Leveille y cols., 2007), enfermedades de la vista (van der Aa y cols., 2013), etc., mientras que se destacan algunos como ejemplos del segundo rubro: el estrés crónico (Peavy y cols., 2012), la tendencia al aislamiento social (Cavanaugh y Poon, 1989), la desmotivación para realizar tareas domésticas cotidianas (Herzog, Dixon, y Hultsch, 1990; Jiang y Xu, 2013), la mayor dependencia para realizar actividades sencillas (Brown, Kiely, Bharel, y Mitchell, 2013), trastornos del sueño (Jaussent y cols., 2011), depresión y trastornos de ansiedad (Feola y cols., 2013).

Uno de los primeros modelos de intervención dirigido a la prevención y disminución de los efectos devastadores del envejecimiento fue la "gerontología conductual" (Baltes y Barton, 1979), un modelo que puede ser adaptado para el tratamiento individual o grupal en AM internos en casas de asistencia, cuyo propósito principal es mejorar y prolongar un estilo de vida independiente entre los AM (Baltes, 1988; Izal 
y Montorio, 1999). En esta misma línea, la revisión de Olazarán y colaboradores (2010), pone de manifiesto la importancia que tienen las diversas estrategias agrupadas en los tratamientos no farmacológicos destinados a mejorar los trastornos cognitivos como la EA en los AM. Destacando que en la implementación de las diversas intervenciones solas o combinadas, como el entrenamiento cognitivo, el ejercicio físico o las actividades lúdicas, se deben tomar en consideración la capacitación y profesionalización de los cuidadores, y en donde además del éxito terapéutico ampliamente referido con la implementación de estos programas, se debe considerar la gran ventaja que representa el bajo costo económico que se requiere para la instrumentación de estas estrategias en los países subdesarrollados.

Estudios recientes han revelado la importancia del entrenamiento combinado (ejercicios físicos y cognitivos), en la obtención de grandes beneficios para adultos mayores sanos y para aquellos que manifiestan bajo nivel de disfunción cognitiva (O’Hara y cols., 2007; Pichierri y cols., 2012).

Algunos estudios han enfatizado la importancia de realizar ejercicio físico con regularidad por parte de AM para disminuir el riesgo en el desarrollo temprano de alteraciones cognitivas (Dik, Deeg, Visser y Jonker, 2003), mientras que otras investigaciones coinciden en exponer los beneficios que brindan para la cognición, tanto las rutinas aeróbicas como las no-aeróbicas (Colcombe y Kramer, 2003). Además, se ha reportado que el ejercicio aeróbico en los AM ejerce diferencias significativas sobre el grado de satisfacción psicológica, posiblemente relacionado con los cambios positivos en el estado de ánimo (de Gracia y Marco, 2000). Consecuentemente, los beneficios del ejercicio aeróbico además de prevenir o frenar el deterioro cognitivo, fortalece el grado de independencia en los AM.

Por todo lo anterior, el propósito principal del presente estudio fue conocer el impacto del PEC en las habilidades cognitivas, la estabilidad emocional y el grado de independencia en una población de adultos mayores institucionalizados (AMI).

\section{Método}

\section{Participantes}

Los ancianos participantes en este estudio viven en la "Casa Hogar Ancianos" (casa de retiro) de Tuxtla Gutiérrez, en el estado de Chiapas; adscrita al organismo público descentralizado conocido como Sistema para el Desarrollo Integral de la Familia (DIF - Chiapas).

El estudio incluyó 23 hombres y 19 mujeres cuya edad oscilaba entre el rango de los 70 a 97 años; (ver la tabla 1). La mayoría de los AMI tenía un nivel socioeconómico bajo, el grado educativo presentado también fue bajo; más de la mitad (56\%) era analfabeta, 15 de ellos $(36 \%)$ completó únicamente la educación primaria, dos $(4.7 \%)$ terminaron la secundaria y tres (7\%) manifestaron haber concluido la preparatoria. La mayor parte de las personas que habitan este asilo ha sido abandonada por sus familiares y sufre de diferentes grados de discapacidad física y/o cognitiva.

Los ancianos con algún deterioro en la salud mental o física que imposibilitaran su evaluación fueron excluidos de este estudio, así como aquellos que se encontraban fuera del rango de edad descrito en el párrafo anterior. El estudio fue instrumentado 
de enero a diciembre de 2011 con los 42 ancianos que dieron su consentimiento previo, y se respaldó con la autorización del Comité de Ética de la Facultad de Medicina Humana de la Universidad Autónoma de Chiapas.

Tabla 1. Distribución de la población de estudio por sexo y edad.

\begin{tabular}{|c|c|c|}
\hline SEXO & $n \cdot(\%)$ & $\begin{array}{c}\text { EDAD } \\
\text { Media } \pm \text {.D.T. }\end{array}$ \\
\hline Hombres & 23.(55) & $79.69 \cdot \pm \cdot 6.60$ \\
\hline Mujeres & 19.(45) & $81.05 \cdot \pm \cdot 7.55$ \\
\hline Total & 43.(100) & $80.31+ \pm \cdot 6.99$ \\
\hline
\end{tabular}

\section{Variables e instrumentos de evaluación}

En el desarrollo de la investigación se evaluaron las siguientes variables: Niveles de ansiedad. Esta evaluación se realizó a través de la prueba de Hamilton (1959) y consiste en 13 ítems relacionados con los signos y síntomas vinculados con el padecimiento, además de una evaluación de la conducta realizada durante la entrevista. El resultado puede fluctuar entre los 0 y los 56 puntos. El obtener un resultado de 6 a 14 puntos indica la necesidad de proporcionar atención clínica al sujeto.

También se evaluaron los signos de depresión, mediante la prueba de Beck (Beck, Epstein, Brown, y Steer, 1988), la cual consiste en 21 ítems; un resultado que va de los 10 a los 18 puntos indica la necesidad de proporcionar atención clínica.

Se valoró la capacidad cognitiva a través Mini-examen cognoscitivo de Lobo y cols. (1999), el cual estaba integrado por cinco áreas de conocimiento: orientación, atención, concentración, planeación, memoria y lenguaje. Este test es utilizado para monitorear a pacientes con demencia y consiste en 35 reactivos con un marcador máximo de 35 . Un resultado de 24 o menor es considerado un indicativo de demencia.

Finalmente, la prueba de Barthel (Cid- Ruzafa y Damián-Moreno, 1997) fue aplicada para estimar el nivel de dependencia de los participantes.

\section{Procedimiento: programa y evaluación}

Se aplicó un programa de entrenamiento combinado (PEC) que incluía ejercicios físicos y cognitivos, técnicas de motivación y actividades orientadas a mejorar las habilidades sociales, así como la capacidad de resolver retos de la vida cotidiana.

El entrenamiento cognitivo se basó en ejercicios del Grupo de trabajo para la estimulación cognitiva de los adultos mayores, desarrollado por Sardinero (2010), que hace énfasis en seis áreas de trabajo: atención, función ejecutiva, lenguaje, memoria, percepción, lectoescritura y construcción visual. Este entrenamiento cognitivo fue combinado con ejercicios aeróbicos de bajo impacto y con estimulación de las funciones motoras a través de varios objetos.

Los participantes llevaron a cabo actividades recreativas dirigidas a mejorar sus habilidades sociales; realizaron ejercicios relacionados con su vida cotidiana, orientados a fortalecer su capacidad de independencia; al mismo tiempo que se incrementó su motivación a través de distintas técnicas conductuales, como el modelado y el reforzamiento positivo. Todas estas prácticas se desarrollaron de forma holística en sesiones 
de dos horas, tres días a la semana, durante doce meses (ver tabla 2). Con la finalidad de observar posibles diferencias con la instrumentación del PEC, se aplicó la batería de pruebas arriba mencionada, al inicio y al final de esta intervención.

\section{Resultados}

Analizamos los resultados en los AMI (mujeres y hombres) con los que se realizó la intervención.

Tabla 2. Actividades que integran al PEC

\begin{tabular}{|l|c|}
\hline \multicolumn{1}{|c|}{ Actividades } & Duración \\
\hline Orientación de la realidad (fecha, hora, etc.) & 20 min. \\
\hline $\begin{array}{l}\text { Actividad sensorial-motora (estiramiento, ejercicio de bajo impacto físico y } \\
\text { la relajación con música). }\end{array}$ & 35 min. \\
\hline $\begin{array}{l}\text { Ejercicios de: atención, concentración y funciones ejecutivas (memori- } \\
\text { zar listas de utensilios cotidianos, el lenguaje, sopa de letras, juegos de do- } \\
\text { minó, rompecabezas, secuencias, categorizaciones, integridad de datos, etc.) } \\
\text { Estimulación sensorial (identificación de objetos, olores, sabores, sonidos, } \\
\text { formas, texturas, etc.) }\end{array}$ & 45 min. \\
\hline \begin{tabular}{l} 
Retroalimentación y despedida. \\
\hline
\end{tabular} & 20 min. \\
\hline
\end{tabular}

\section{Análisis de datos}

Dadas las características de la muestra y de las variables estudiadas se utilizaron los siguientes procedimientos estadísticos: Distribución de frecuencias para la variable sexo; puntuaciones medias para la variable edad; test de Wilcoxon para muestras relacionadas, para comparaciones entre variables evaluadas en un mismo sujeto en distintos momentos, y prueba U de MannWhitney para dos muestras independientes, orientada a analizar las diferencias entre los hombres y las mujeres tanto al inicio como al final del PEC.
Grupo de AMI mujeres: Los resultados obtenidos por los 19 individuos que participan correctamente en el PEC se muestran en la siguiente tabla (Tabla 3). Podemos observar que en la totalidad de las variables estudiadas no se advierten diferencias significativas al comparar los resultados obtenidos de las pruebas psicológicas aplicadas al inicio y al final del PEC.

Grupo de los AMI hombres: El impacto positivo en la mayoría de las variables estudiadas se apreció de manera sorprendente en este grupo (23 hombres); el PEC redujo de manera significativa tanto los niveles de 
ansiedad como de depresión, e incrementó la capacidad de independencia. Se pudo apreciar de manera significativa la disminución de la depresión en este grupo, cuando se contrastó con los niveles de ansiedad de las mujeres en el periodo posterior a la intervención. No obstante, por lo que a las capacidades cognitivas se refiere, se mantuvieron estables durante el desarrollo del estudio, de acuerdo con la medición efectuada.

Lo anterior demuestra la eficiencia del Programa de entrenamiento combinado PEC tanto para mejorar como para mantener el funcionamiento de las distintas áreas neuropsicológicas, en los adultos mayores.

\section{Discusión}

Como ya se ha señalado, la dificultad del AM para adaptarse y mantener la homeostasis en la vejez depende de diversos factores biopsicosociales; por ejemplo: afrontar problemas de tensión o estrés, ante diversas situaciones como: el aislamiento, la pérdida de familiares y amigos, la inseguridad económica, las enfermedades crónicas y la cercanía de la muerte (Brenes y cols., 2005; 2007); en donde, la capacidad para sobreponerse a los agentes estresantes está relacionada con la estructura genética, el medio ambiente en el que se desarrolla el individuo, el grado de funcionalidad de los diversos aparatos y sistemas del organismo,

Tabla 3. Diferencias de medias de cada una de las variables antes (Pre) y después (Post) de la intervención.

\begin{tabular}{|c|c|c|c|c|c|c|c|c|}
\hline \multirow[t]{2}{*}{ VARIABLES } & \multicolumn{2}{|c|}{$\begin{array}{l}\text { Pre } \\
m(D T)\end{array}$} & \multicolumn{2}{|c|}{$\begin{array}{l}\text { Post } \\
\text { m(DT) }\end{array}$} & \multicolumn{2}{|c|}{$\begin{array}{l}\text { Wilcoxon } \\
\text { Z (p) }\end{array}$} & \multicolumn{2}{|c|}{$\begin{array}{l}\text { U de Mann-Whitney } \\
U(p)\end{array}$} \\
\hline & M & $H$ & M & $H$ & $\begin{array}{l}\text { M Pre vs } \\
\text { M Post }\end{array}$ & $\begin{array}{l}\text { H Pre vs } \\
\text { H Post }\end{array}$ & M Pre vs $\mathrm{H}$ Pre & $\begin{array}{l}\text { M Post vs } \\
\text { H Post }\end{array}$ \\
\hline Ansiedad & $\begin{array}{l}15.10 \\
(6.81)\end{array}$ & $\begin{array}{c}18.34 \\
(10.74)\end{array}$ & $\begin{array}{l}14.52 \\
(5.93)\end{array}$ & $\begin{array}{l}14.30 \\
(5.32)\end{array}$ & $\begin{array}{l}0.09 \\
\text { (n.s) }\end{array}$ & $\begin{array}{c}2.16 \\
\left({ }^{*} p<0.03\right)\end{array}$ & 241 (n.s) & 208 (n.s) \\
\hline Depresión & $\begin{array}{l}15.68 \\
(6.35)\end{array}$ & $\begin{array}{l}19.30 \\
(9.76)\end{array}$ & $\begin{array}{l}18.53 \\
(9.07)\end{array}$ & $\begin{array}{l}12.69 \\
(6.10)\end{array}$ & 1.09 (n.s) & $\begin{array}{c}2.38 \\
\left({ }^{*} p<0.01\right)\end{array}$ & 266.5 (n.s) & $\begin{array}{c}133 \\
\left({ }^{*} p<0.03\right)\end{array}$ \\
\hline Capacidad Cognitiva & $\begin{array}{l}17.63 \\
(9.56)\end{array}$ & $\begin{array}{l}17.01 \\
(9.37)\end{array}$ & $\begin{array}{l}16.10 \\
(9.30)\end{array}$ & $\begin{array}{l}16.13 \\
(7.78)\end{array}$ & 1.76 (n.s) & 92.5 (n.s) & 210.5 (n.s) & 213.5 (n.s) \\
\hline Independencia & $\begin{array}{c}57.05 \\
(32.93)\end{array}$ & $\begin{array}{c}50.21 \\
(36.51)\end{array}$ & $\begin{array}{c}59.94 \\
(32.71)\end{array}$ & $\begin{array}{c}55.13 \\
(31.52)\end{array}$ & 0.66 (n.s) & $\begin{array}{c}2.03 \\
\left({ }^{*} p<0.05\right)\end{array}$ & 190.5 (n.s) & 200.5 (n.s) \\
\hline
\end{tabular}

Nota. m=media; DT=Desviación típica; H=hombres; M=mujeres 
así como de la eficacia con que estos sistemas dan respuesta a las situaciones estresantes (Briones y cols., 2012; Oitzl, Champagne, van der Veen, y de Kloet, 2010). En esta misma línea, se ha reportado que los AMI tienen problemas médicos y psicológicos más graves que los AM que permanecen con sus familias (Hyer y Blazer, 1982; Rodríguez, 2007); además, el estrés puede tener una influencia marcada tanto en el desarrollo de trastornos afectivos como en la esperanza de vida (Oitzl y cols., 2010).

En el mismo contexto, referente a las instituciones de los AM en países subdesarrollados como México, factores como la inadecuada infraestructura, la nula profesionalización del personal para el cuidado de los mismos, carencia de tratamientos médicos integrales, podrían representar verdaderas fuentes de estrés (Ruipérez y Llorente, 2004), que también generan un impacto negativo sobre las áreas afectivas y cognitivas de los AMI (Velasco, Mendoza, Torres, y Saucedo, 2011).

Dentro de los estados afectivos, la ansiedad tiene una relación estrecha con el estrés, ya que ambos estados comparten similar regulación por diversos sistemas de neurotransmisión (Briones-Aranda, López, y Picazo, 2002; Kapfhammer, 2011) y presentan interacción en sus manifestaciones clínicas (Kapfhammer, 2011). La relación estrés-ansiedad se evidencia claramente en los AMI (Eaton y cols., 2011) y la prevalencia es mayor en los AM que viven en el medio urbano (Prina, Ferri, Guerra, Brayne, y Prince, 2011).

En la actualidad existe una variedad de estrategias encaminadas a disminuir los efectos negativos de la ansiedad en los AMI. Por ejemplo, la implementación tanto de un programa de ejercicio físico (Casas-
Herrero y Izquierdo, 2012) como de un PEC han impactado en el decremento de la ansiedad de los AMI (Rebok, Carlson, y Langbaum, 2007). Lo anterior está en línea con el impacto positivo del PEC sobre la disminución en la ansiedad que se observó en el grupo de los hombres. Sin embargo en el grupo de las mujeres, posterior a la implementación del PEC, los niveles de ansiedad subclínica se mantuvieron constantes con respecto a los niveles obtenidos antes de la intervención, lo cual coincide con los resultados obtenidos en otros estudios en donde se ha referido que los AMI pueden mantener niveles de ansiedad leve o subclínica (Fisher y Noll, 1996; Sheikh y Cassidy, 2000).

Por otra parte, es bien conocida la disminución significativa de los estrógenos en mujeres tanto a nivel sérico como cerebral en el periodo postmenopáusico (Bixo, Backstrom, Winblad y Andersson1995; Rosario y cols., 2011; Barron y Pike, 2012). De manera contraria, otros estudios en hombres han referido niveles conservados de testosterona circulantes en los AM (Muller y cols., 2003; Hogervorst, 2013), a pesar de la reducciones de esta hormona conforme aumenta la edad; e incluso otra investigación ha reportado que el principal metabolito activo de la testosterona, la hidrotestosterona, no parece tener cambios con la edad (Kaufman y Vermeulen, 2005). Por todo lo anterior y considerando la importancia de los estrógenos en los trastornos de ansiedad y depresión ampliamente referidos en estudios con animales y humanos (Picazo, Estrada-Camarena y Hernández-Aragón, 2006; Walf y Frye, 2006; Studd, 2011), es posible sugerir, que el factor hormonal podría estar influyendo en las diferencias encontradas, después de la implementación 
del PEC, en los niveles de ansiedad y depresión de las mujeres cuando se compararon con las hombres, a pesar de que en nuestra población de estudio no se midieron las hormonas esteroideas gonadales a nivel sérico.

Por otra parte, dentro de las alteraciones del estado de ánimo ligadas a eventos estresantes crónicos que suelen presentar los AM se encuentra la depresión (Durá, Stukenberg, y Kiecolt-Glaser, 1990). La prevalencia de la depresión en la población geriátrica oscila entre 10 a $20 \%$ (Barua Ghosh, Kar, y Basilio, 2010). Los datos epidemiológicos muestran que los AMI presentan mayor prevalencia de depresión en comparación con la población de AM no institucionalizados (de Menezes y Bachion, 2008). Además las mujeres tienen mayor tendencia a padecer cuadros depresivos que los hombres (Bekker y van Mens-Verhulst, 2007; Prina y cols., 2011). Uno de los factores implicado en la etiología de los trastornos del estado de ánimo y la ansiedad, es el aumento en la capacidad de respuesta al estrés (Carvalho-Netto y cols., 2011). Lo que induce a proponer la teoría de la vulnerabilidad al estrés, centrada en tratar de explicar la tendencia que tienen las mujeres a padecer en mayor proporción depresión y ansiedad (Almaida y cols., 2012; Chou y Cheung, 2013). Sin embargo, actualmente no se han encontrado evidencias contundentes coherentes con las diferencias en las reacciones para lidiar con el estrés entre hombres y mujeres (Ong, Phillips y Chai, 2013), además existe relativamente poco conocimiento acerca de tales diferencias de género entre los AM (Ong y cols., 2013).

Por lo anterior, es indispensable continuar estudiando en el futuro esta propuesta, con la finalidad de tener más elementos que permitan ayudar a encontrar mejores explicaciones relacionadas con nuestros resultados.

Otro factor a considerar en el análisis de la depresión, es lo descrito en varios estudios, que correlacionan el bajo nivel escolar de los AM con la presencia de trastornos depresivos (Bojorquez-Chapela y cols., 2009; Li y cols., 2013; Jozwiak, Preville, y Vasiliadis, 2013), por lo que es muy factible que la mayor proporción de analfabetismo observada en el grupo de las mujeres, en comparación con los hombres (datos no incluidos), haya influido de manera contundente tanto en el efecto positivo del PEC sobre la reducción de la depresión en los hombres, como en la falta de efecto del PEC sobre los niveles de depresión en el grupo de las mujeres. Sin embargo, el hecho de que en las mujeres no se observen cambios significativos en los niveles de depresión, no demerita la influencia positiva del PEC sobre esta área afectiva, ya que al igual que con la ansiedad la ejecución del PEC mantuvo uniformes las manifestaciones de depresión en las mujeres, después de doce meses de la intervención.

Por otra parte, el déficit cognitivo puede anteceder a una demencia y es multifactorial, ya que se ha relacionado tanto con el estrés crónico como con los factores asociados a los trastornos afectivos. Por lo anterior, al tratar de esquematizar la compleja vinculación entre los trastornos afectivos (ansiedad y depresión) y el deterioro cognitivo, se puede ilustrar a través de una disposición cíclica de los elementos afectivos, en donde la influencia del estrés incida en el centro del círculo e influya sobre la homeostasis interna de cada organismo.

Por todo lo anterior, la posible explicación de la aparente falta de efecto del PEC so- 
bre el deterioro cognitivo en ambos sexos, podría estar basada en el efecto sumatorio de los factores estresantes generados en el medio institucional previamente mencionados, que se contraponen e incluso superan los efectos beneficiosos del PEC.

No obstante, nuevamente es rescatable el mantenimiento en el puntaje obtenido en la mayoría de las pruebas aplicadas en comparación con los niveles observados en la línea base; por lo cual en otros estudios, este hallazgo es considerado como un éxito terapéutico (Izal y Montorio, 1999).

En suma a lo antepuesto, se podría considerar al PEC como una estrategia adecuada para evitar el detrimento en la capacidad cognitiva de los AMI, similar a lo que ha sido reportado en otros estudios con otros PEC (Rebok y cols., 2007).

Por otro lado, la capacidad de independencia es proporcional al grado de funcionalidad física y neuropsicológica de los AM, en donde el padecer depresión y ansiedad hacen más vulnerable al AM para generar dependencia (Horgas, Wilms y Baltes, 1998). En el presente estudio, en los AMI (mujeres), se encontraron niveles de dependencia moderados que se mantuvieron sin cambios a lo largo de la implementación del PEC. El argumento para tratar de explicar estos hallazgos, pudiera estar relacionado con estudios previos, en donde los trastornos depresivos y la ansiedad en los AM disminuyeron la capacidad de independencia para hacer frente a las actividades de la vida diaria (Grabovich y cols., 2010; Schustser, Manetti, Aeschimann, y Limosin 2013). Además, estos trastornos pueden influir negativamente en el desarrollo adecuado de algunos programas de rehabilitación, específicamente en la falta de capacidad para aprender nuevas tareas, en el procesamiento de la información y la orientación al logro de determinados objetivos (Horowitz, Reinhardt, Boerner, y Travis, 2003; Lenze y cols., 2007). Además, de otros factores relacionados con los cuidadores, por ejemplo, el impedir a los AMI la realización de casi toda actividad cotidiana (aseo personal, desarrollo de habilidades, traslado peatonal, etc.), aunado a la ausencia de motivación hacia el logro; elementos que se relacionan con la teoría de las contingencias (Maier y Seligman, 1975). De manera contraria, en los hombres se constató la eficacia del PEC, a lo largo de su instrumentación, caracterizado por un aumento en la capacidad de independencia, esta diferencia posiblemente también esté relacionada con el efecto positivo que ejerció el PEC sobre los niveles de ansiedad y depresión en los AMI hombres.

Por último, actualmente en el Estado de Chiapas, México, únicamente se cuenta con actividades lúdicas o deportivas aisladas en las instituciones encargadas atender a los AM y se carece de un PEC que coadyuve en el mejoramiento de la salud mental. Así, nuestro objetivo de cara al futuro es continuar en la profundización de las estrategias para la aplicación del PEC en grupos de estudio similares y contribuir en mejorar la integración y perfeccionamiento de este programa. Lo anterior, basado en las propiedades del PEC que atiende, fortalece e impacta, las tres esferas de los AM: física, psicológica y social, detonando en el fortalecimiento del AM para enfrentar los embates negativos del estrés, para disminuir los trastornos afectivos, evitar el progreso del deterioro cognitivo, lo que redundara en el mejoramiento de la independencia y la calidad de vida los AMI, a bajo costo. 


\section{Referencias}

Almeida, 0. P., Draper, B., Pirkis. J., Snowdon, J., Lautenschlager, N. T., Byrne, G., Sim, M., Stocks, N., Kerse, N., Flicker, L., y Pfaff, J. J. (2012). Anxiety, depression, and comorbid anxiety and depression: risk factors and outcome over two years. International psychogeriatrics /IPA, 24 (10), 1622-1632.

Alosco, M. L., Brickman, A. M., Spitznagel, M. B., van Dulmen, M., Raz, N., Cohen, R., Sweet, L. H., Colbert, L. H., Josephson, R., Hughes, J., Rosneck, J., y Gunstad, J. (2012). The independent association of hypertension with cognitive function among older adults with heartfailure. Journal of the neurological sciences, 323 (1-2), 216-20.

Ballesteros, S., Reales, J. M., Mayas, J., y Heller, M. A. (2008). Selective attention modulates visual and haptic repetition priming: effects on ageing and Alzheimer's disease. Experimental Brain Research, 189 (4), 473-483.

Ballesteros, S., y Reales, J. M. (2004). Intact haptic priming in normal aging and Alzheimer's disease: evidence for dissociable memory systems. Neuropsychologia, 42 (8), 1063-1070.

Baltes, M. M. (1988). The etiology and maintenance of dependency in the elderly: Three phases of operant research. Behavior Therapy, 19, 301-319.

Baltes, M. M., y Barton, E. (1979). Behavioral analysis of aging: A review of the operant model and research. International Journal of Behavioral Development, 2, 297-320.

Barret, T. R., y Watkins, S. K. (1986). Word familiarity and cardiovascular health as determinants of age-related recall differences. Journal of Gerontology, 41 (2), 222-224.

Barron, A. M., y Pike, C. J. (2012). Sex hormones, aging, and Alzheimer's disease. Frontiers in bioscience (Elite edition), 4, 976-997.

Barua, A., Ghosh, M. K., Kar, N., y Basilio, M. A. (2010). Socio-demographic Factors of Geriatric Depression. Indian Journal Psychological Medicine, 32 (2), 87-92.

Beck, A. T., Epstein, N., Brown, G., y Steer, R. A. (1988). An Inventory for measuring clinical anxiety: Psychometric Properties. Journal of Consulting and Clinical Psychology, 56 (6), 893-897.

Bekker, M. H., y van Mens-Verhulst, J. (2007). Anxiety disorders: sex differences in prevalence, degree, and background, but gender-neutral treatment. Gender Medicine, 4, 178-193.

Bixo, M., Backstrom, T., Winblad, B., y Andersson, A. (1995). Estradiol and testosterone in specific regions of the human female brain in different endocrine states. The
Journal of steroid biochemistry and molecular biology, 55 (3-4), 297-303.

Bojorquez-Chapela, I., Villalobos-Daniel, V. E., ManriqueEspinoza, B. S., Tellez-Rojo, M. M., y Salinas-Rodríguez, A. (2009). Depressive symptoms among poor older adults in Mexico: prevalence and associated factors. Pan American journal of public health, 26 (1), 70-77.

Brenes, G. A., Guralink, J. M., Wiliamison, J. D., Fried, L. P., Simpson, C., Simonsick, E. M., y Penninx, B. W. (2005). The influence of anxiety on the progression of disability. Journal American Geriatric Society, 53 (1), 34-39.

Brenes, G. A., Kritchevsky, S. B., Mehata, K. M., Yaffe, K., Simonsick, E. M., Ayonayon, H. N., Rosano, C., Rubin, S. M., Satterfield, S., y Penninx, B. W. (2007). Scared to death: results from the health, Aging and Body, Composition study. Journal American GeriatricSociety, 15 (3), 262-265.

Briones, A., Gagno, S., Martisova, E., Dobarro, M., Aisa, B., Solas, M., Tordera, R., y Ramírez, M. (2012). Stressinduced anhedonia is associated with an increase in Alzheimer's disease-related markers. British Journal Pharmacological, 165 (4), 897-907.

Briones, A., López, C., y Picazo, 0. (2002). Influence of forced swimming-induced stress on the anxiolytic-like effect of 5 HT (1A) agents in mice. Psychopharmacology, $162(2), 147-155$.

Brown, R.T., Kiely, D.K., Bharel, M., y Mitchell, S.L. (2013). Factors associated with geriatric syndromes in older homeless adults. Journal of health care for the poor and underserved, 24(2), 456-468.

Carvalho-Netto, E. F., Myers, B., Jones, K., Solomon, M. B., Herman, J. P. (2011). Sex differences in synaptic plasticity in stress-responsive brain regions following chronic variable stress. Physiology and behavior, 104 (2), 242-247.

Casas-Herrero, A., y Izquierdo, M. (2012). Physical exercise as an efficient intervention in frail elderly persons. Anales Del Sistema Sanitario De Navarra, 35 (1), 69-85.

Cavanaugh, J. C., y Poon, L. W. (1989). Metamemorial Predictors of memory performance in young and older adults. Psychology and Aging, 4 (3), 365-368.

Chou, K. L, Cheung, K. C. (2013). Major depressive disorder in vulnerable groups of olderadults, their course and treatment, and psychiatric comorbidity. Depress Anxiety, 30 (6), 528-537.

Cid-Ruzafa, J., y Damián-Moreno, J. (1997). Valoración de la discapacidad física: el índice de Barthel. Revista Española de Salud Pública, 71 (2), 127-137.

Colcombe, S., y Kramer, A. F. (2003). Fitness effects on the cognitive function of older adults: a meta - analytic study. Psychological Science, 14 (2), 125-130. 
de Gracia, M., y Marco, M. (2000). Efectos psicológicos de la actividad física en personas mayores. Psicothema, 12 (2), 285-292.

deMenezes, R. L., y Bachion, M. M. (2008). Study of intrinsic risk factors for falls in institutionalized elderly people. Ciência\&SaúdeColetiva, 13 (4):1209-1218.

Dik, M., Deeg, D. J., Visser, M., y Jonker, C. (2003). Early life physical activity and cognition at old age. Journal of Clinical and Experimental Neuropsychology, 25 (5), 643653.

Dura, J. R., Stukenberg, K. W., y Kiecolt-Glaser, J. K. (1990). Chronic stress and depressive disorders in older adults. Journal Abnormal Psychology, 99 (3), 284-290.

Eaton, N. R., Krueger, R. F., Keyes, K. M., Skodol, A. E., Markon, K. E., Grant, B. F., y Hasin, D. S. (2011). Borderline personality disorder co-morbidity: relationship to the internalizing-externalizing structure of common mental disorders. Psychological Medicine, 41 (5), 1041-1050.

Eaton, W. W., y Keyl, P. M. (1990). Risk factors for the onset of Diagnostic Interview Schedule/DSM-III agoraphobia in a prospective, population-based study. Archives of General Psychiatry, 47 (9), 819-824.

Feola, M., Garnero, S., Vallauri, P., Salvatico, L., Vado, A., Leto, L., y Testa, M. (2013). Relationship between Cognitive Function, Depression/Anxiety and FunctionalParameters in Patients Admitted for Congestive Heart Failure. The open cardiovascular medicine journal, 7, 54-60.

Fisher, J. E., y Noll, J. P. (1996). Anxiety disorders. In L. L. Carstensen, B. A. Edelstein, y L. Dornbrand (Eds.) The practical handbook of clinical gerontology (pp. 304-323). Thousand Oaks, CA: Sage.

Grabovich, A., Lu, N., Tang, W., Tu, X., y Lyness, J. M. (2010) Outcomes of subsyndromaldepression in older primary care patients. The American journal of geriatric psychiatry : official journal of the American Association for Geriatric Psychiatry, 18 (3), 227-235.

Grenier, S., Préville, M., Boyer, R., O'Connor, K., Béland, S. G., Potvin, O., Hudon, C., y Brassard, J. (2011). The impact of DSM-IV symptom and clinical significance criteria on the prevalence estimates of subthreshold and threshold anxiety in the older adult population. The American Journal of Geriatric Psychiatry, 19 (4), 316-326.

Hamilton, M. (1959). The assessment of anxiety states by rating. British Journal Medicine Psychology, 32 (1), 50-55.

Herzog, C., Dixon, R. A., y Hultsch, D. F. (1990). Relationships between metamemory, memory predictions and memory task performance in adults. Psychology and Aging, 5 (2), 215-227.

Hogervorst, E. (2013). Effects Of Gonadal Hormones On Cognitive Behavior In Elderly Men And Women. Journal of neuroendocrinology, doi: 10.1111/jne.12080.
Horgas, A. L., Wilms, H. U., y Baltes, M. M. (1998). Daily life in very old age: everyday activities as expression of successful living. The Gerontologist, 38 (5), 556-568.

Horowitz, A., Reinhardt, J. P., Boerner, K., y Travis, L. A. (2003). The influence of health, social support quality and rehabilitation on depression among disabled elders. Aging \& mental health, 7 (5), 342-350.

http://www.inegi.org.mx/est/contenidos/proyectos/estadistica/default.aspx

Hyer, L., y Blazer, D. G. (1982). Depressive symptoms: impact and problems in long term care facilities. International Journal of Behavioral Geriatrics, 1 (3), 33-44.

Izal, M., y Montorio, I. (1999). Gerontología Conductual, Bases para la Intervención y ámbitos de aplicación. España: (Ed.) Síntesis.

Jaussent, I., Dauvilliers, Y., Ancelin, M. L., Dartigues, J. F., Tavernier, B., Touchon, J.,Ritchie, K., yBesset, A. (2011). Insomnia symptoms in older adults: associated factors andgender differences. The American journal of geriatric psychiatry: official journal of the American Association for Geriatric Psychiatry, 19 (1):88-97.

Jiang, C., y Xu, Y. (2013). The association between mild cognitive impairment and doing housework. Aging MentHealth, PMID: 23919266.

Jozwiak, N., Preville, M., y Vasiliadis, H. M. (2013). Bereavement-related depression in the older adult population: A distinct disorder? Journal of affective disorders, doi:pii: S0165-0327(13)00672-1. 10.1016/j.jad.2013.08.038.

Kapfhammer, H. P. (2011). The relationship between depression, anxiety and heart disease- a psychosomatic challenge. Psychiatric Danubina, 23(4), 412-424.

Kaufman, J. M., y Vermeulen, A. (2005). The decline of androgen levels in elderly men and its clinical and therapeutic implications. Endocrine reviews, 26(6), 833-876.

Lenze, E. J., Skidmore, E. R., Dew, M. A., Butters, M. A., Rogers, J. C., Begley, A., Reynolds, C. F. 3rd, y Munin M. C. (2007). Does depression, apathy or cognitive impairment reduce the benefit of inpatient rehabilitation facilities for elderly hip fracture patients? General hospital psychiatry, 29 (2), 141-146.

Leveille, S. G., Bean, J., Ngo, L., McMullen, W., yGuralnik J. M. (2007). The pathway frommusculoskeletal pain to mobility difficulty in older disabled women. Pain, 128(12), 69-77.

Li, D., Zhang, D. J., Shao, J. J., Qi, X. D., y Tian, L. (2013). A meta-analysis of the prevalence of depressive symptoms in Chinese older adults. Archives of gerontology and geriatrics, doi:pii: S0167-4943(13)00135-0. 10.1016/j. archger.2013.07.016.

Light, L. L. (1991). Memory and aging: four hypotheses in 
search of data. AnnualReviewPsychology, 42, 333-376.

Lobo, A., Saz, P., Marcos, G., Día, J. L., de la Cámara, C., Ventura, T., Morales Asín, F., Fernando Pascual L., Montañés J. A., y Aznar S. (1999). Revalidation and standardization of the cognition mini-exam (first Spanish version of the Mini-Mental Status Examination) in the general geriatric population. Medicina Clínica (Barcelona), 112 (20), 767-774.

Maier, S. F., y Seligman, M. E. (1975). Learned helplessness: Theory and evidence. Journal of Experimental Psychology, 105 (1), 3-46.

Muller, M., den Tonkelaar, I., Thijssen, J. H., Grobbee, D. E., y van der Schouw, Y. T. (2003). Endogenous sex hormones in men aged, 40-80 years. European journal of endocrinology / European Federation of Endocrine Societies, 149(6), 583-589.

O’Hara, R., Brooks, J. 0., Friedman, L., Schöder, C. M., Morgan, K. S., y Kraemer, H. C. (2007). Long-term effects of mnemonic training in community-dwelling older adults. Journal of Psychiatric Research, 41 (7), 585-590.

Oitzl, M. S., Champagne, D. L., van der Veen, R., y de Kloet, E. R. (2010). Brain development under stress: hypotheses of glucocorticoid actions revisited. Neuroscience \& Biobehavioral Reviews, 34 (6), 853-866.

Olazarán, J., Reisberg, B., Clare, L., Cruz, I., Peña-Casanova, J., Del Ser, T., Woods, B., Beck, C., Auer, S., Lai, C., Spector, A., Fazio, S., Bond, J., Kivipelto, M., Brodaty, H., Rojo, J. M., Collins, H., Teri, L., Mittelman, M., Orrell, M., Feldman, H. H., y Muñiz, R. (2010). Nonpharmacological therapies in Alzheimer's disease: a systematic review of efficacy. Dementia and geriatric cognitive disorders, 30 (2), 161-178.

Ong, F. S, Phillips, D. R., Chai, S. T. (2013). Life events and stress: do older men and womenin Malaysia cope differently as consumers? Journal of cross-cultural gerontology, 28 (2), 195-210.

Peavy, G. M., Jacobson, M. W., Salmon, D. P., Gamst, A. C., Patterson, T. L., Goldman, S., Mills, P. J., Khandrika, S., Galasko, D. (2012). The influence of chronic stress on dementia-relateddiagnostic change in older adults. Alzheimer disease and associateddisorders, 26 (3), 260-266.

Peraita, H., y Moreno, F.J. (2006). Análisis de la estructura conceptual de categorías semánticas naturales y artificiales en una muestra de pacientes de Alzheimer. Psicothema, 18 (3), 492-500.

Peraita, H., y Sánchez Bernardos, M. L. (1998). Evaluación del deterioro de diversos aspectos de la memoria semántica en pacientes con Demencia tipo Alzheimer. Anales de Psicología, 14(2), 193-206.

Pérez, G. (2006). Intervención y Desarrollo Integral en personas mayores. Madrid: (Ed.) Universitas.
Picazo, 0., Estrada-Camarena, E., y Hernandez-Aragon, A. (2006). Influence of the post-ovariectomy time frame on the experimental anxiety and the behavioural actions of some anxiolytic agents. European journal of pharmacology, 530 (1-2), 88-94.

Pichierri, G., Coppe, A., Lorenzetti, S., Murer, K., de Bruin, E.D. (2012). The effect of a cognitive-motor intervention on voluntary step execution under single and dual task conditions in older adults: a randomized controlled pilot study. Clinical interventions in aging, 7, 175-184.

Prina, A. M., Ferri, C. P., Guerra, M., Brayne, C., y Prince, M. (2011). Prevalence of anxiety and its correlates among older adults in Latin America, India and China: crosscultural study. The British Journal of Psychiatry, 199 (6), 485-491.

Rao, A. A. (2013). Views and opinion on BDNF as a target for diabetic cognitivedysfunction. Bioinformation, 9 (11), 551-554.

Rebok, G. W., Carlson, M. C., y Langbaum, J. B. (2007). Training and maintaining memory abilities in healthy older adults: traditional and novel approaches. The Journal of Gerontology B Psychological Sciences Social Sciences, 62 (1), 53-61.

Rodríguez, P. (2007). Residencias y otros alojamientos para personas mayores. España: (Ed.) Médica Panamericana.

Rosario, E. R., Chang, L., Head, E. H., Stanczyk, F. Z., y Pike, C. J. (2011). Brain levels of sex steroid hormones in men and women during normal aging and in Alzheimer's disease. Neurobiology of aging, 32, 604-613.

Ruipérez, I., y Llorente, P. (2004).Guía para Auxiliares y Cuidadores del Anciano. España: (Ed.) McGraw Hill.

Sardinero, A. (2010). Estimulación Cognitiva para Adultos: Cuadernos de trabajo. Madrid España: (Ed.) Grupo Gesfomedia.

Schuster, J. P., Manetti, A., Aeschimann, M., yLimosin F. (2013). [Epidemiology of psychiatric disorders in elderly and their impact on somatic health]. Gériatrie et psychologie neuropsychiatrie du vieillissement, 11 (2), 181-185.

Sheikh, J. I., y Cassidy, E. L. (2000). Treatment of anxiety disordersin the elderly: issues and strategies. Journal of Anxiety Disorders, 14 (2), 173-190.

Soares C. N., y Frey B. N. (2010). Challenges and opportunities to manage depression during the menopausal transition and beyond. Psychiatric Clinics of North America, 33 (2), 295-308.

Studd, J. W. (2011). A guide to the treatment of depression in women by estrogens. Climacteric, 14 (6), 637-642.

Van der Aa, H. P., van Rens, G.H., Comijs, H.C., Bosmans, J.E., Margrain, T.H., y van Nispen, R.M. (2013). Stepped- 
care to prevent depression and anxiety in visually impaired older adults-design of a randomised controlled trial. BMC Psychiatry, 13, 209.

Velasco, R., Mendoza, R. C., Torres, M. M., y Saucedo, M. D. (2011). Estado funcional, mental y social del adulto mayor institucionalizado de colima. México, Universidad de Colima.

Walf, A. A., y Frye, C. A. (2006). A review and update of mechanisms of estrogen in the hippocampus and amygdala for anxiety and depression behavior. Neuropsychopharmacology, 31 (6), 1097-1111.

Fecha de recepción: 17/04/2013

Fecha de aceptación: 25/11/2013 